\title{
An Assessment of Hand Erosive Osteoarthritis: Correlation of Radiographic Severity with Clinical, Functional and Laboratory Findings
}

\author{
Fabio Massimo Perrotta - Silvia Scriffignano - Antonia De Socio • \\ Ennio Lubrano (D)
}

Received: January 9, 2019 / Published online: February 6, 2019

(C) The Author(s) 2019

\begin{abstract}
Introduction: The present study aimed (a) to evaluate the clinical and radiographic characteristics of hand erosive osteoarthritis (EOA) in a group of consecutive patients, (b) to correlate the severity of radiographic involvement with clinical and laboratory findings and (c) to associate the levels of pain and functional impairment with some radiographic findings.

Methods: Patients with EOA were consecutively enrolled. Inclusion criteria required the American College of Rheumatology (ACR) criteria for hand osteoarthritis and the presence of at least one joint in " $E$ " or " $\mathrm{R}$ " phase according to Verbruggen-Veys. For each patient, demographic and clinical data were collected including evaluation of pain and function with the Australian Canadian Osteoarthritis Hand Index (AUSCAN) scale and Health Assessment Questionnaire (HAQ). Laboratory parameters and plain radiography of both hands were also collected. Each radiograph was evaluated in accordance with
\end{abstract}

Enhanced digital features To view enhanced digital features for this article go to https://doi.org/10.6084/ m9.figshare.7660793.

F. M. Perrotta - S. Scriffignano - A. De Socio ·

E. Lubrano $(\square)$

Dipartimento di Medicina e Scienze, della salute

"Vincenzo Tiberio", Università degli Studi del

Molise, Campobasso, Italy

e-mail: enniolubrano@hotmail.com the Verbruggen-Veys classification and scored with the Kallman score.

Results: During the study period 60 patients (M/F 13:47) with EAO were enrolled. More severe radiographic disease ("E" or " $\mathrm{R}$ ") was often found at II and III distal interphalangeal (IP) joints. In addition, Kallman score, presence of osteophytes, erosions and joint space narrowing correlated significantly with duration of symptoms, AUSCAN, pain and active joints. More severe radiographic involvement was associated with AUSCAN and with the presence of ankylosis only at proximal IP joints.

Conclusion: The present study showed that EOA is characterised by a significant correlation between radiographic involvement and some clinical characteristics of the disease. However, an impairment of joint function was mainly associated to radiological proximal IP joint involvement, but not with other symptoms such as pain.

Keywords: Clinical assessment; Erosive; Imaging; Osteoarthritis; Outcome

\section{INTRODUCTION}

Osteoarthritis (OA) of the hand is a highly prevalent disease. Typically, radiographic features of hand OA can be found in $67 \%$ of women and $55 \%$ of men, aged 55 years or older [1], while symptomatic hand $\mathrm{OA}$ is less 
common. In people older than 70 years, prevalence of $26.2 \%$ in women and $13.4 \%$ in men have been reported [2]. Erosive osteoarthritis (EOA) is considered a more aggressive subset of hand $\mathrm{OA}$, with the main involvement of proximal and distal interphalangeal (IP) finger joints. It was first described by Crain [3] who introduced the concept of "inflammatory OA" and after few years by Peter et al. [4] who coined the term EOA. In this peculiar condition, some characteristic features could be identified: osteophyte formation and joint space narrowing are associated with the presence of bone destruction, which can be identified with the presence of erosions on radiographic evaluation. It has been hypothesized, with the support of different evidence from radiological studies, that inflammatory changes in the synovia and subchondral bone may contribute to the development of the disease $[5,6]$.

Osteolytic areas and bone destruction are followed by episodes during which the affected tissues are repaired and remodelled [7]. Clinically, patients affected by the disease present considerable pain and functional limitation. Some authors showed a complex and rapidly progressive disease with the early onset of erosions and joint deformity that might have an impact on hand function. Furthermore, EOA significantly affects the patients' quality of life, working and social activities, with a negative impact on general health of affected subjects comparable with inflammatory arthritis such as rheumatoid arthritis [8]. Moreover, patients with EOA may develop a severe limitation in joint motion with important functional impairment [9].

Although there are different reports on the clinical impact of EOA in terms of pain and articular function, few studies assessed the association of a patient's clinical features with the presence of more severe radiographic disease. Moreover, in EOA, despite the factors that contribute to functional impairment being evaluated in different studies [8-10], the association between localization of lesions and such impairment is still poorly understood. In addition, the question whether the pain and functional impairment experienced by patients with EOA are caused by the presence of aggressive erosive radiographic changes or by the acquired structural damage of the IP joints, due to reparative phenomena, still remains unanswered. Finally, few data are available on the association of the radiographic findings (e.g. proximal or distal IP joints) with pain and function. On the basis of this background, the aim of this study was (a) to evaluate the clinical and radiographic characteristics of hand EOA in a group of consecutive patients, (b) to correlate the severity of radiographic involvement with clinical and laboratory findings and (c) to associate the levels of pain and functional impairment with some radiographic findings.

\section{METHODS}

\section{Study Design}

All patients participating in the present study were evaluated at the Rheumatology Outpatient Clinic of the University of Molise. Between 1 January 2017 and 31 December 2017, patients with EOA were consecutively enrolled. Different definitions of EOA are present in the literature. In our study, EOA was defined by the presence of the American College of Rheumatology (ACR) criteria for OA of hands plus at least one joint in " $\mathrm{E}$ " or " $\mathrm{R}$ " phase according to Verbruggen-Veys [7, 11].

Inclusion criteria were (1) subjects aged 18 years or over, (2) patients fulfilling the ACR criteria for OA of hands [12] plus at least one joint in " $\mathrm{E}$ " or " $\mathrm{R}$ " phase according to Verbruggen-Veys [7], (3) availability of plain radiography of the hands performed within 1 month before the visits, (4) absence of diagnosis or symptoms/signs suggesting other musculoskeletal diseases, (5) absence of rheumatoid factor or anti-citrullinated peptide antibodies. For each patient, at baseline study visit, demographic and clinical data were collected. Age, sex, symptoms duration and body mass index (BMI) were collected. Evaluation of tender and clinically active (swollen) joints was performed. A clinically active joint was defined as tenderness and effusion upon palpation. Presence of reduced range of motion (defined as a reduction of more than $50 \%$ of normal joint motility 
assessed with a goniometer) or joint ankylosis (defined as complete block of articular motion with radiographic evidence of ankylosis), pain and patient global assessment of disease activity (PtGA) on visual analogue scale (VAS), Australian Canadian Osteoarthritis Hand Index (AUSCAN) [13] and the Health Assessment Questionnaire (HAQ) were evaluated. Laboratory parameters (C-reactive protein, erythrocyte sedimentation rate and serum vitamin D) were collected. Comorbidities and current treatment were also evaluated.

\section{Radiographic Assessment}

Plain radiography of both hands performed at least 1 month before the clinical examination was also collected. Distal IP, proximal IP, the first carpometacarpal (CMC) and the trapezioscaphoid (TS) joints of both hands were evaluated on each radiography in accordance with the Verbruggen-Veys classification and scored with the Kallman score [14] by two readers (FMP and ADS), in a blind observation. Disagreements were resolved by a third examiner (EL). Verbruggen-Veys grading classifies each joint in five stages: "N", normal; "S", stationary (only OA findings); "J", joint space disappearance; "E", erosive; "R", remodelling [7]. A more severe radiological involvement of single joint was defined when a stage " $E$ " or " $R$ " was present.

Every patient gave his/her written consent and the study was in accordance with the ethical standards of the responsible committee on human experimentation (institutional and national) and with the Helsinki Declaration of 1975 , as revised in 2000. The study was also approved by the scientific committee of University of Molise (protocol no. 0002-092017).

\section{Statistical Analysis}

Statistical analysis was carried out using the SPSS package (version 17.0, Chicago, IL). After testing for normal distribution of data, we reported descriptive results as mean (standard deviation, SD) or median (interquartile range,
IQR) values for continuous variables or number (percentages) for categorical ones.

The significance of the differences was determined the Mann-Whitney test for unpaired samples for non-normally distributed variables. Categorical variables were compared by $\chi$-square test or Fisher's exact test. Agreement between the readers for the radiographic evaluation was assessed with Cohen's $\kappa$ coefficient.

Spearman correlation coefficient was used to analyse associations among the radiographic Kallman score, the presence of erosions, osteophytes and joint space narrowing with clinical and laboratory data. Univariate analysis was performed to study the association among the function and other clinical variables with the radiographic localization of more severe disease (stage " $\mathrm{E}$ " or " $\mathrm{R}$ ") at either proximal and distal joints.

All statistical procedures were two-sided; a significance level was accepted at $p<0.05$.

\section{RESULTS}

During the study period 60 patients (M/F 13:47) with EOA, fulfilling the inclusion criteria, were enrolled. Mean age (SD) was 66.4 (7.7) years. Clinically active (swollen) joints were present in 35 patients $(58.3 \%)$. Presence of joint with limited range of motion/ankylosis (at least one) was found in 25 patients (41.6\%). Table 1 shows the clinical, laboratory and radiographic features of the enrolled patients. The number of patients with EOA lesions according to Verbruggen-Veys grading is shown in the Fig. 1. Agreement (Cohen's $\kappa$ ) between the two readers was 0.84 . More severe disease (" $E$ " or " $R$ " phase) was often found at II and III distal IP joints and was associated with the presence of clinical ankylosis/limited range of motion. First CMC severe involvement was present in only $6.6 \%$ of patients. The symmetric distribution of lesions was also confirmed. Table 2 shows the correlations of Kallman's radiographic score, the number of erosion at each joint site, the number of osteophytes at each joint site and the presence of joint space narrowing at each joint site with the different clinical and laboratory variables. The Kallman score correlates 
Table 1 Clinical, laboratory and radiographic features of the patients $(n=60)$ with erosive osteoarthritis

Male/female

Mean age $(\mathrm{SD})$, years

Symptoms duration, mean (SD), years

Body mass index, median (IQR)

Smoking (current/past), $n$ (\%)

Pain on VAS (median/IQR)

PtGA on VAS (median/IQR)

Tender joints (median/IQR)

Clinically active (swollen) joints (median/IQR)

Presence of joint with limited range of motion/ankylosis (at least one), $n$ (\%)

HAQ (median/IQR)

AUSCAN, median (IQR) (0-60)

Presence of comorbidity (any), $n$ (\%)

C-reactive protein (median/IQR), mg/L

Serum vitamin D (median/IQR), $\mu \mathrm{g} / \mathrm{L}$

Treatment

Classical NSAIDs (including acetaminophen), $n$ (\%)

COX-2 inhibitors, $n$ (\%)

Glucosamine, $n(\%)$

Chondroitin sulfate, $n(\%)$

Hydroxychloroquine, $n$ (\%)

Kallman radiographic score (median/IQR)
$13: 47$

$66.4(7.7)$

$7.9(5.4)$

$25.48(22.5-29.2)$

$15(25)$

$55(37.5-75.5)$

$31(50-60)$

$4(2-8)$

$1(0-2)$

25 (41.6)

$0.75(0.37-1.06)$

$33.1(21-43)$

$45(75)$

$2(2-4)$

$32.2(15.2-38.9)$

$22(36.7)$

18 (30)

17 (28.3)

8 (13.3)

$3(5)$

$39.5(35-47.5)$

$V A S$ visual analogue scale, $S D$ standard deviation, $I Q R$ interquartile range, $\operatorname{Pt} G A$ patient global assessment, $H A Q$ Health Assessment Questionnaire, AUSCAN Australian Canadian Osteoarthritis Hand Index, NSAIDs non-steroidal anti-inflammatory drugs, $C O X-2$ cyclooxygenase-2

significantly with duration of symptoms, AUSCAN, HAQ and clinically active (swollen) joints but not with the age, tender joints or level of pain. No correlation was also found with laboratory parameters. Interestingly, the presence of erosions correlates only with the presence of pain on VAS, while osteophytes significantly correlate with age, symptoms duration, pain on VAS, tender joints and active joints, HAQ and AUSCAN. As shown in Fig. 1, prevalence of more severe radiographic involvement ("E" or
" $\mathrm{R}$ " phase) was higher in the distal IP joints; however, more severe radiographic involvement ("E" or "R" phase) in proximal IP but not at distal IP joints was associated with higher AUSCAN, HAQ values and the presence of joints with limited range of motion/ankylosis as shown in Table 3. No other association between localization of lesions and the other clinical and laboratory parameters was found. 

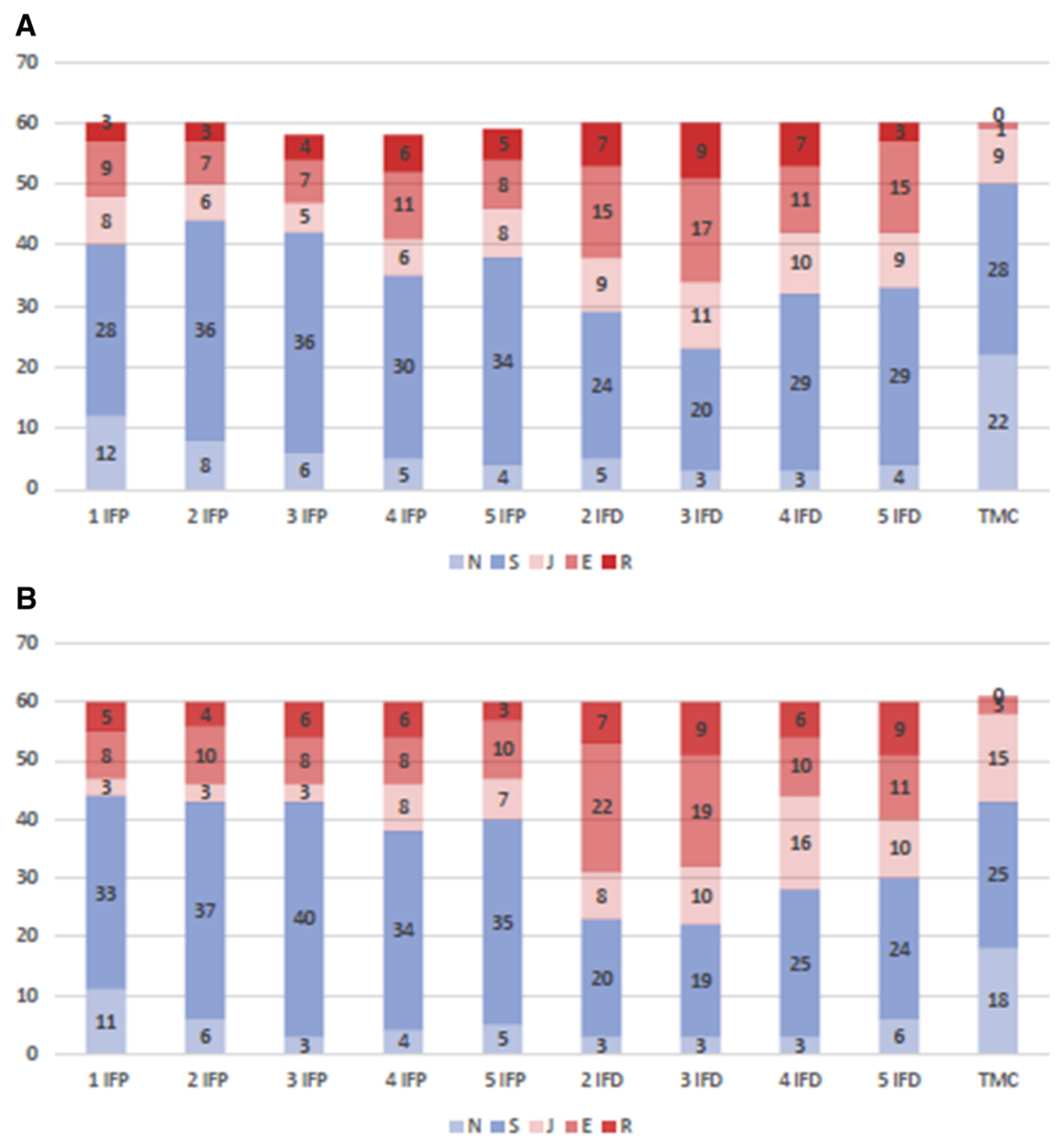

Fig. 1 Number of patients with each $x$-ray finding according to Verbruggen-Veys in the right (a) and left (b) hand. N normal, $\mathrm{S}$ stationary (only OA findings with

\section{DISCUSSION}

EOA is characterized by destructive lesions affecting the IP joints, suggesting a pivotal role of inflammation and bone resorption as shown by both presence of signs of inflammation in the synovial membrane of patients with OA and presence of erosions [15-17]. However, despite some similarities with inflammatory arthritis, EOA joints experienced a unique sequence of destructive/remodelling events [7]. Our study confirmed the presence of significant correlation between radiographic involvement and clinical burden of disease and, in particular, bony growth seems to be correlated with space narrowing), J joint space disappearance, E presence of erosions, $\mathrm{R}$ remodelling

clinical symptoms such as pain and function. Different studies have previously shown that patients with EOA experience more pain and functional limitation than patients with nonerosive OA, with worst hand mobility, and were less satisfied with hand function and aesthetics $[18,19]$. Other studies demonstrated that patients with hand OA experience at least as much hand pain as patients with RA [19]. Finally, patients with EOA have more functional impairment and significantly more pain compared to patients with controlled inflammatory arthritis affecting the hands [20]. This highlights the significant clinical burden of EOA and warrants the search for new treatment 
Table 2 Correlation of Kallman's score, number of erosion at each joint site, number of osteophytes at each joint site and presence of joint space narrowing at each joint site with the different clinical and laboratory variables

\begin{tabular}{|c|c|c|c|c|c|c|c|c|}
\hline & \multicolumn{2}{|c|}{ Kallman score } & \multicolumn{2}{|l|}{ Erosions } & \multicolumn{2}{|c|}{ Osteophytes } & \multicolumn{2}{|l|}{$\begin{array}{l}\text { Joint space } \\
\text { narrowing }\end{array}$} \\
\hline & $\begin{array}{l}\text { Spearman } \\
(\rho)\end{array}$ & $P$ value & $\begin{array}{l}\text { Spearman } \\
(\rho)\end{array}$ & $P$ value & $\begin{array}{l}\text { Spearman } \\
(\rho)\end{array}$ & $P$ value & $\begin{array}{l}\text { Spearman } \\
(\rho)\end{array}$ & $P$ value \\
\hline Age & 0.22 & NS & 0.15 & NS & 0.27 & 0.04 & 0.23 & NS \\
\hline Body mass index & 0.02 & NS & 0.12 & NS & 0.01 & NS & 0.01 & NS \\
\hline Symptoms duration & 0.41 & 0.001 & 0.20 & NS & 0.45 & 0.001 & 0.39 & 0.003 \\
\hline Pain on VAS & 0.16 & NS & 0.17 & NS & 0.27 & 0.04 & 0.14 & NS \\
\hline PtGA on VAS & 0.12 & NS & 0.15 & NS & 0.1 & NS & 0.11 & NS \\
\hline Tender joints & 0.17 & NS & 0.27 & 0.04 & 0.26 & 0.05 & 0.1 & NS \\
\hline $\begin{array}{l}\text { Clinically active (swollen) } \\
\text { joints }\end{array}$ & 0.34 & 0.01 & 0.03 & NS & 0.43 & 0.001 & 0.35 & 0.009 \\
\hline HAQ & 0.30 & 0.02 & 0.13 & NS & 0.40 & 0.002 & 0.27 & 0.04 \\
\hline AUSCAN & 0.33 & 0.01 & 0.20 & NS & 0.42 & 0.01 & 0.24 & NS \\
\hline C-reactive protein, $\mathrm{mg} / \mathrm{L}$ & 0.11 & NS & 0.02 & NS & 0.06 & NS & 0.11 & NS \\
\hline
\end{tabular}

$V A S$ visual analogue scale, $P t G A$ patient global assessment, HAQ Health Assessment Questionnaire, AUSCAN Australian Canadian Osteoarthritis Hand Index, NS not significant

Table 3 Association (odd ratio/95\% CI) of pain on VAS, presence of joints with limited range of motion/ankylosis, function (AUSCAN) and quality of life (HAQ) with the localization of more severe disease ( $E$ ” or “ $R$ ” phase)

\begin{tabular}{|c|c|c|c|c|}
\hline & \multicolumn{2}{|l|}{$\begin{array}{l}\text { Proximal IP joint } \\
\text { involvement }\end{array}$} & \multicolumn{2}{|c|}{ Distal IP joint involvement } \\
\hline & $\begin{array}{l}\text { Odd ratio }(95 \% \\
\text { CI })\end{array}$ & $P$ value & $\begin{array}{l}\text { Odd ratio }(95 \% \\
\text { CI })\end{array}$ & $P$ value \\
\hline Pain in VAS $(>50 \mathrm{~mm}$ vs $\leq 50 \mathrm{~mm})$ & $1.3(0.45-4.05)$ & NS & $1.9(0.4-8.9)$ & NS \\
\hline $\begin{array}{l}\text { Joints with limited range of motion/ankylosis (presence vs } \\
\text { absence) }\end{array}$ & $4.34(1.34-14.3)$ & 0.01 & $1.33(0.28-6.26)$ & NS \\
\hline $\operatorname{AUSCAN}(>30$ vs $\leq 30)$ & $5.33(1.59-17.8)$ & 0.001 & $4.94(0.89-27.31)$ & NS \\
\hline HAQ $(>0.5$ vs $\leq 0.5)$ & $4.54(1.37-15.07)$ & 0.01 & $3.02(0.63-14.38)$ & NS \\
\hline
\end{tabular}

$V A S$ visual analogue scale, AUSCAN Australian Canadian Osteoarthritis Hand Index, HAQ Health Assessment Questionnaire

strategies [8]. In fact, the treatment of EOA is mainly based on the reduction of pain with non-steroidal anti-inflammatory drugs and bisphosphonate agents, and there is no strong evidence for disease-modifying effects of different compounds such as hydroxychloroquine, methotrexate, biologic drugs, glucosamine and chondroitin sulfate [21-23]. The knowledge of 
clinical and radiographic characteristics associated with pain and function could potentially be useful to understand what kind of long-term treatment strategies have to follow. In our study we demonstrated that, although the prevalence of more severe lesions was higher in distal IP joints, reduced function assessed by AUSCAN and reduced range of motion/ankylosis are mainly associated with proximal IP involvement. This is not surprising because hand function and hand activities are mainly supported by proximal IP joints. However, no other association was found, in particular with the level of pain, that seems to be independent from the anatomical site involved, although pain is an important aspect to consider in EOA, as it was found to be associated with higher risk of further radiographic progression [10]. Furthermore, the distribution of lesions is in keeping with previous reports [24]. These results are also in keeping with a previous report in hand $\mathrm{OA}$, in which discordance between radiographic OA scores and both pain and functional impairment was observed [25]. In our study, disability and quality of life were not associated with a more severe involvement of distal IP which, in turn, should be taken into account for the disease management. Furthermore, another interesting result was the correlation between the clinical and laboratory parameters with radiographic lesions. In our study, the presence of osteophytes significantly correlates with a number of variables including age, symptoms duration, pain on VAS, tender joints and active joints, HAQ and AUSCAN, suggesting that bone growth may be an important determinant of clinical burden of the disease, whereas erosions were weakly associated only with the level of pain. The results of our study seem to suggest that treatment strategies in the near future might directly act on the inhibition of bone growth, even if further studies are needed. On the other hand, longitudinal studies showed that pain, tenderness, soft tissue swelling, "J" and " $E$ " phase were significantly associated with erosive progression in hand OA, suggesting that even these factors together with bony growth should be taken into account in the assessment of disease [10]. Finally, in our report, the clinical and radiographic involvement of other hand joints (such as metacarpophalangeal and carpometacarpal joints) apart from IP was found to be mild, as reported elsewhere, and seems to not be associated with function and pain $[26,27]$.

This work showed some limitations: first, this report was designed as a cross-sectional study and no information is available on possible factors associated with radiographic progression. Second, the number of patients enrolled was relatively small; however, despite this potential weakness, the study could provide useful information about the burden of disease in EOA.

\section{CONCLUSIONS}

Our study confirmed the presence of significant correlation between radiographic involvement and some clinical features of disease and, in particular, bony growth seems to be correlated with clinical symptoms and function. Moreover, an impairment of joint function seems to be related to proximal joint involvement rather than distal involvement, but other symptoms such as pain are independent from the anatomical site involved. Finally, it might be interesting, in future studies, to complete this kind of evaluation with other imaging techniques, such as magnetic resonance or ultrasonography.

\section{ACKNOWLEDGEMENTS}

We thank the participants of the study.

Funding. No funding or sponsorship was received for this study or publication of this article. All authors had full access to all of the data in this study and take complete responsibility for the integrity of the data and accuracy of the data analysis.

Authorship. All named authors meet the International Committee of Medical Journal Editors (ICMJE) criteria for authorship for this article, take responsibility for the integrity of the work as a whole, and have given their approval for this version to be published. 
Authorship Contributions. All authors should have made substantial contributions to all of these sections: conception and design of the study, acquisition of data, analysis and interpretation of data, drafting the article, revising it critically for important intellectual content and final approval of the version to be submitted.

Disclosures. Fabio Massimo Perrotta, Silvia Scriffignano, Antonia De Socio and Ennio Lubrano have nothing to disclose.

Compliance with Ethics Guidelines. Every patient gave his/her written consent and the study was in accordance with the ethical standards of the responsible committee on human experimentation (institutional and national) and with the Helsinki Declaration of 1975 , as revised in 2000. The study was also approved by the scientific committee of University of Molise (protocol no. 0002-09-2017)

Data Availability. The datasets analyzed during the current study are available from the corresponding author on reasonable request.

Open Access. This article is distributed under the terms of the Creative Commons Attribution-NonCommercial 4.0 International License (http://creativecommons.org/licenses/ by-nc/4.0/), which permits any noncommercial use, distribution, and reproduction in any medium, provided you give appropriate credit to the original author(s) and the source, provide a link to the Creative Commons license, and indicate if changes were made.

\section{REFERENCES}

1. Dahaghin S, Bierma-Zeinstra SM, Ginai AZ, Pols HA, Hazes JM, Koes BW. Prevalence and pattern of radiographic hand osteoarthritis and association with pain and disability (the Rotterdam study). Ann Rheum Dis. 2005;64:682-7.

2. Neogi T. The epidemiology and impact of pain in osteoarthritis. Osteoarth Cartil. 2013;21:1145-53.
3. Crain DC. Interphalangeal osteoarthritis. JAMA. 1961;175:1049-53.

4. Peter JB, Pearson CM, Marmor L. Erosive osteoarthritis of the hands. Arthritis Rheum. 1966;9:365-88.

5. Kwok WY, Kloppenburg M, Rosendaal FR, Van Meurs JB, Hofman A, Bierma-Zeinstra SM. Erosive hand osteoarthritis: its prevalence and clinical impact in the general population and symptomatic hand osteoarthritis. Ann Rheum Dis. 2011;70:1238-42.

6. Punzi L, Favero M, Frallonardo P, Ramonda R. Time to redefine erosive osteoarthritis. RMD Open. 2015;1:e000105.

7. Verbruggen G, Veys EM. Numerical scoring systems for the anatomic evolution of osteoarthritis of the finger joints. Arthritis Rheum. 1996;39:308-20.

8. Wittoek R, Cruyssen BV, Verbruggen G. Predictors of functional impairment and pain in erosive osteoarthritis of the interphalangeal joints: comparison with controlled inflammatory arthritis. Arthritis Rheum. 2012;64:1430-6.

9. Ter Borg EJ, Bijlsma JW. Spontaneous ankylosis in erosive osteoarthritis of the finger joints: a case series of eight postmenopausal women. Clin Rheumatol. 2014;33:1015-7.

10. Meersseman P, Van De Vyver C, Verbruggen G, Elewaut D, Wittoek R. Clinical and radiological factors associated with erosive radiographic progression in hand osteoarthritis. Osteoarth Cartil. 2015;23:2129-33.

11. Gazeley DJ, Yeturi S, Patel PJ, Rosenthal AK. Erosive osteoarthritis: a systematic analysis of definitions used in the literature. Semin Arthritis Rheum. 2017;46:395-403.

12. Altman R, Alarcon G, Appelrouth D, et al. The American College of Rheumatology criteria for the classification and reporting of osteoarthritis of the hand. Arthritis Rheum. 1990;33:1601-10.

13. Poole JL. Measures of hand function: arthritis Hand Function Test (AHFT), Australian Canadian Osteoarthritis Hand Index (AUSCAN), Cochin Hand Function Scale, Functional Index for Hand Osteoarthritis (FIHOA), Grip Ability Test (GAT), Jebsen Hand Function Test (JHFT), and Michigan Hand Outcomes Questionnaire (MHQ). Arthritis Care Res (Hoboken). 2011;63(Suppl 11):S189-99.

14. Kallman DA, Wigley FM, Scott WWJR, Hochberg $\mathrm{MC}$, Tobin JD. New radiographic grading scales for osteoarthritis of the hand: reliability for 
determining prevalence and progression. Arthritis Rheum. 1989;32:1584-91.

15. Kortekaas MC, Kwok WY, Reijnierse M, Huizinga TW, Kloppenburg M. In erosive hand osteoarthritis more inflammatory signs on ultrasound are found than in the rest of hand osteoarthritis. Ann Rheum Dis. 2013;72:930-4.

16. Schraml C, Schwenzer NF, Martirosian P, et al. Assessment of synovitis in erosive osteoarthritis of the hand using DCE-MRI and comparison with that in its major mimic, the psoriatic arthritis. Acad Radiol. 2011;18:804-9.

17. Favero $M$, Perino $G$, Valente ML, Tiengo $C$, Ramonda R. Radiological and histological analysis of two replaced interphalangeal joints with active subchondral bone resorption in erosive hand osteoarthritis: a novel mechanism? Skelet Radiol. 2017;46:385-91.

18. Bijsterbosch J, Watt I, Meulenbelt I, Rosendaal FR, Huizinga TW, Kloppenburg M. Clinical burden of erosive hand osteoarthritis and its relationship to nodes. Ann Rheum Dis. 2010;69:1784-8.

19. Maheu E, Michon M, Carrat F, Berenbaum F. Erosive versus non-erosive hand OA: prospective crosssectional comparison of clinical data [abstract]. Osteoarth Cartil. 2008;16(Suppl 4):R292.

20. Slatkowsky-Christensen B, Mowinckel P, Kvien TK. Health status and perception of pain: a comparative study between female patients with hand osteoarthritis and rheumatoid arthritis. Scand J Rheumatol. 2009;38:342-8.

21. Persson MSM, Sarmanova A, Doherty M, Zhang W. Conventional and biologic disease-modifying anti- rheumatic drugs for osteoarthritis: a meta-analysis of randomized controlled trials. Rheumatology (Oxford). 2018;57:1830-7.

22. Aitken D, Laslett LL, Pan F, et al. A randomised double-blind placebo-controlled crossover trial of HUMira (adalimumab) for erosive hand OsteoaRthritis-the HUMOR trial. Osteoarth Cartil. 2018;26:880-7.

23. Saviola G, Abdi-Ali L, Povino MR, Campostrini L, Sacco S, Carbonare LD. Intramuscular clodronate in erosive osteoarthritis of the hand is effective on pain and reduces serum COMP: a randomized pilot trial-The ER.O.D.E study (ERosive Osteoarthritis and Disodium-clodronate Evaluation). Clin Rheumatol. 2017;36(10):2343-50.

24. Addimanda O, Mancarella L, Dolzani P, et al. Clinical and radiographic distribution of structural damage in erosive and nonerosive hand osteoarthritis. Arthritis Care Res (Hoboken). 2012;64(7):1046-53.

25. Pattrick M, Aldridge S, Hamilton E, Manhire A, Doherty M. A controlled study of hand function in nodal and erosive osteoarthritis. Ann Rheum Dis. 1989;48:978-82.

26. Kwok WY, Kloppenburg M, Marshall M, Nicholls E, Rosendaal FR, Peat G. The prevalence of erosive osteoarthritis in carpometacarpal joints and its clinical burden in symptomatic community-dwelling adults. Osteoarth Cartil. 2014;22:756-63.

27. Addimanda O, Cavallari C, Pignotti E, et al. Radiographic involvement of metacarpophalangeal and radiocarpal joints in hand osteoarthritis. Clin Rheumatol. 2017;36:1077-82. 